\title{
Adverse Drug Reactions to Disulfiram Treatment with or without Alcohol Challenge in the Indian Setting: Systematic Review
}

\author{
1Jaison Joseph, ${ }^{2}$ Debasish Basu
}

\begin{abstract}
Background: Disulfiram has been used for treating alcohol dependence in the Indian population for the last three decades. The safety concerns associated with adverse effectsraise a high index of suspicion for disulfiram therapy in Indian population. Surprisingly few reviews exist on adverse reaction to disulfiram treatment and most of such articles published during the 1990s. Moreover, such systematic investigation relevant to the Indian population is missing in the literature. Thepresent review aims to narrate and discuss disulfiram induced adverse reactionsin the Indian setting.
\end{abstract}

Materials and methods: We analyzed clinical trials, observational studies and case reports of adverse drug reactions to disulfiram therapy reportedin the Indian setting. Relevant studies published within 2017 in the electronic databases: Medline, IndMED, Ermed, and Embase were included. The Naranjo adverse drug reaction probability scale (NADRPS) was used for assessing adverse drug reactions to disulfiram treatment.

Results: The present review identified four open-label trials, four observational studies and 24 case reports describing thirtytwo cases of disulfiram-induced adverse reactions in the Indian setting. Psychiatric adverse events and de novo convulsions were the common adverse reactions to disulfiram treatment without alcohol challenge in this setting. The NADRPS score was found to be from 5-9 and the magnitude of the adverse effects associated with disulfiram-ethanol reaction was largely associated with surreptitious use.

Conclusion: The review is a pioneer attempt for an evidence base summary of common adverse reactions and temporal association of dose-dependent adverse effects to disulfiram treatment in the Indian setting. The results of the review open an area of discussion regarding ethnic variations and the adverse effects of disulfiram in clients treated for alcohol dependence in this setting.

Keywords: Adverse drug reaction, Disulfiram, India.

How to cite this article: Joseph J, Basu D. Adverse Drug Reactions to Disulfiram Treatment with or without Alcohol Challenge

\footnotetext{
${ }^{1}$ Lecturer, ${ }^{2}$ Professor

${ }^{1}$ College of Nursing, Postgraduate Institute of Medical Sciences, Rohtak, Haryana, India

${ }^{2}$ Drug De-addiction and Treatment Centre, Department of Psychiatry, Postgraduate Institute of Medical Education and Research, Chandigarh, India

Corresponding Author: Jaison Joseph, Lecturer, College of Nursing, Post Graduate Institute of Medical Sciences, Rohtak, Haryana, India. Phone: 07205476571, e-mail: jaisonjsph@ yahoo.com
}

in the Indian Setting: Systematic Review. J Postgrad Med Edu Res 2019;53(1):21-30.

Source of Support: Nil

Conflict of Interest: None

\section{BACKGROUND}

The burden and problems due to alcohol dependence are well documented at the global level. ${ }^{1}$ Disulfiram is the oldest and the first medication approved by FDA for treating alcohol dependence. ${ }^{2}$ It is a well-known empirically tested deterrent agent that produces a disulfiramethanol reaction (DER) for those who concomitantly use alcohol and disulfiram due to the accumulation of acetaldehyde. DER may develop within 5-15 minutes of alcohol intake and include flushing, sweating, nausea, vomiting, palpitations, dyspnoea, tremors, confusion, restlessness, drowsiness, and hypotension. ${ }^{3}$ The general side effects of disulfiram in the absence of alcohol consumption are a headache, general weakness, and dizziness which may subside within weeks of the onset of therapy. ${ }^{4}$ Disulfiramhas been used for treating alcohol dependence in the Indian population for the last three decades. ${ }^{5-12}$ Although found to be efficacious, disulfiram is no longer considered as first-line treatment for alcohol dependence due to difficulties with compliance and toxicity $^{13-15}$ Treatment adherence to disulfiram is notably affected in India due to its common surreptitious use by mixing in food items with the agreement of caregivers or sold as traditional medicines by faith healers. ${ }^{16,17}$ The safety concerns associated with the adverse effects of disulfiram therapy raises a high index of suspicion of its use in Indian population. ${ }^{18}$ The exploration of the pre and post disulfiram treatmentstatusmay help to fill the lacuna regarding the temporal association of adverse reactions to disulfiram treatment. Surprisingly few reviews exist on adverse reaction to disulfiram treatmentwhich was published during the 1990s. ${ }^{19}$ Moreover, such systematic investigation relevant to the Indian population is missing in the literature.

\section{OBJECTIVES}

The objectives of the present study are to narrate and discuss the systematic evidence regarding adverse reactions to disulfiram treatment with or without alcohol challenge in the Indian setting. 


\section{MATERIALS AND METHODS}

We analyzed clinical trials, observational studies and case reports related to adverse drug reactionsto disulfiram therapy reported in the Indian setting.Adverse drug reactions to disulfiram treatment are more reported as case reports as compared to other hierarchy of evidence. Therefore, the CAse REport (CARE) guidelines-a consensus-based clinical case report guideline was used for standardization of case reports. ${ }^{20,21}$ Disulfiram-induced adverse drug reactions were rated based on NADRPS scores. NADRPS is a simple and widely used scale that categorizes the severity of adverse drug reactions as follows: definite if the overall score is 9 or greater, probably for a score of 5 to 8 , possible for 1 to 4 , and doubtful if the score is $0 .^{22}$

\section{Search Strategy}

Relevant studies conducted in the Indian setting and published up to 2017 in the electronic databases: Medline, IndMED, Ermed, and Embase were included. The search strategy used the following keywords: "disulfiram" "alcohol dependence" "adverse drug reaction" "case report" "safety" and "Indian setting".

- Selection criteria: We included studies and case reports published in the English language based on the following criteria: (a) disulfiram as a primary medication for producing adverse effects with or without alcohol challenge (b) adverse effects associated with disulfiram reported in the Indian setting. Disulfiram reactions associated with other substances such as cocaine were excluded in this review. A pre-designed data extraction scheme was used for screening and retrieval of data and all the references with insufficient details were requested in full text. Studies were initially selected based on information from title and abstract and the potentially relevant references were reviewed for possible inclusion and duplicates were excluded. An extensive evaluation of the relevant articles was carried out and assessed for final inclusion.

\section{Data Extraction}

Data extraction scheme included the following aspects: author, disulfiram dosage regimen, disulfiram pre and post-treatment status, major findings and NADRPS score. Both the authors independently searched and selected the potentially relevant studies. Data extraction was jointly carried out and further reviewed as per desired guidelines.

\section{RESULTS}

\section{Study Selection}

The search strategy identified 567 relevant references which we electronically screened and 161 were retrieved for detailed evaluation. Of these, 61 were controlled and cross-sectional studies conducted at the global level. Finally, 38 unique studies including case reports met the inclusion criteria for the review. Figure 1 provides the sequence of the inclusion process of references in the systematic review.

\section{Description of Studies}

\section{Open-label Trials}

Four open-label trails conducted among358 alcoholdependent men found that disulfiram was superior in preventing alcohol relapse as compared to naltrexone, acamprosate and topiramate treatment. Disulfiram was administered in a daily dose of $250 \mathrm{mg}$ for a period of 26-52 weeks, and the commonly observed adverse effects were nausea, drowsiness, abdominal pain, and diarrhea. All the reported adverse effects were minimal in severity and disappeared within the 1-2 week of the initiation of disulfiram treatment (De Sousa; 2004, 2005, $2008,2009){ }^{23-26}$

\section{Observational Studies}

The review identified one observational study that evaluated the treatment response to carefully administered disulfiram to a selected population of 75 subjects with alcohol challenge. Disulfiram was administered in a loading dose of $1500 \mathrm{mg}-2500 \mathrm{mg}$ per day in divided

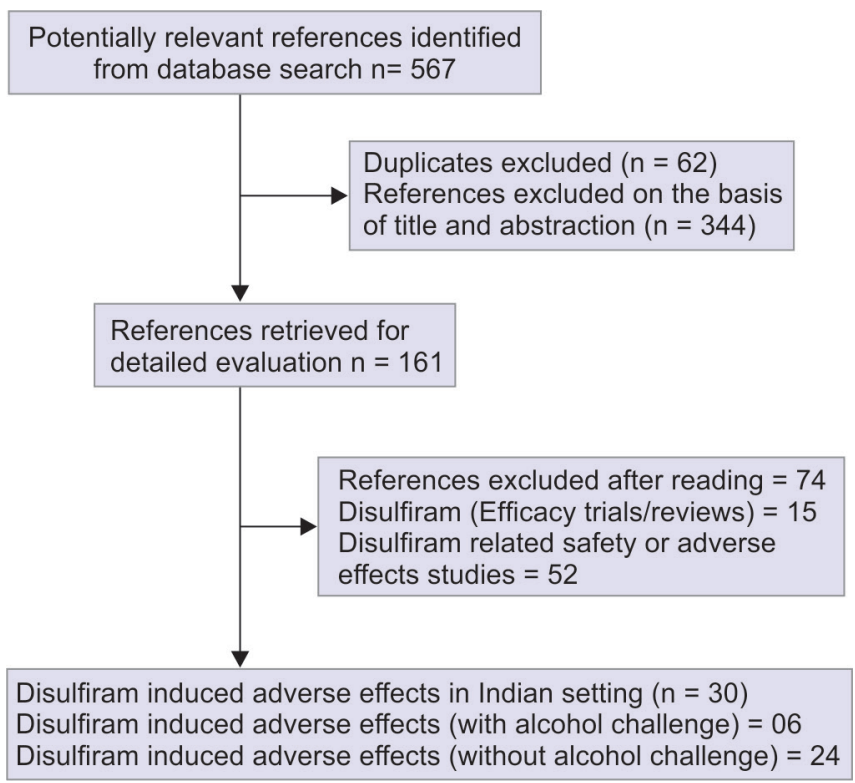

Fig. 1: Flow chart of the inclusion process of references in the systematic review 
doses with a challenging maximum dose of $90 \mathrm{~mL}$ alcohol. The study reported psychotic episodes and skin reactions $(n=3)$ as the side effect of disulfiram treatment which was disappeared within 2 to 3 days of discontinued medication (Bagadia et al.). ${ }^{27}$

Three studies explored the adverse effects of disulfiram treatment (125-500 mg per day) among 261 subjects within a period of one to 2 months. In that two of the studiesnoted psychiatric reactions as major adverse effect to disulfiram treatment. However further analysis found that these symptoms were present before taking the drug and was aggravated with disulfiram treatment (Srinivasan et al., Murthy). ${ }^{28,29}$ The fourth study found few adverse drug reaction to disulfiram treatment such as gastrointestinal symptoms, skin reactions, anxiety and depression to a daily dosage regimen of $125 \mathrm{mg}$ (Paltty et al.). ${ }^{30}$

\section{Case Studies}

The present review identified thirty-two cases of disulfiram-induced adverse reactions with and without alcohol challenge in the Indian setting. ${ }^{31-54}$ Most of the case reports were adequate with the quality of information as per 13 items checklist in the CARE guidelines. The failure to add the word "case report" in the title (Item-I) was the only major missingcomponent as per CARE guidelines in the included case studies $(n=18)$. Descriptions of the included case reports are summarized as per characteristics of cases such as author, disulfiram dosage regimen, disulfiram pre- and post-treatment status, major findings and NADRPS score (Tables 1 and 2).

\section{Disulfiram-Induced Adverse Drug Reactions without Alcohol Challenge}

There are 26 cases describing disulfiram-induced adverse drug reactions without alcohol challenge, and the mean age of the participant is 36 years $(S D=7)$. Most of the clients were taking 250-500 mg disulfiram tablets, and adverse effects were reported within one week to 3 months of the initiation of therapy. Most of the clients were dependent users of alcohol for 4 to 10 years and nine of them were co-morbid dependent users of alcohol and tobacco. Disulfiram was found to be the probable causal factor for adverse effects as the NADRPS Score was found to be from 5-9. All the adverse effects were reduced or completely resolved by stopping or reducing the dose of disulfiram. Adverse reactions to disulfiram treatment as observed in the case reports are described below.

\section{Psychiatric Adverse Events}

The onset of the symptoms of psychosis was reported within 3 to 4 weeks of disulfiram use in four cases with oral intake of up to $500 \mathrm{mg}$ disulfiram. The delusion of persecution and auditory hallucination were the major symptoms of psychosis and recovery was reported within one to two weeks of stoppage of disulfiram.

Four case reports were available on disulfiram induced catatonia in which the age of the clients was ranged from 28-54 years. Symptoms of catatonia such as mutism, rigidity, posturing were reported within 36-72 hours of administration of 500 and $2500 \mathrm{mg}$ of disulfiram in two of the cases. None of the cases reported any organic etiology for developing catatonia but two cases were having the previous history of disulfiram-induced catatonia. Most of the catatonic symptoms resolved within 3-7 days of discontinuation of disulfiram with or without specific management such as injection lorazepam.

Two case reports of clients with alcohol dependence syndrome reported delirium like reactions such as fluctuating sensorium, self-muttering behavior, altered sleepwake cycle following 48 hours to 8 weeks of disulfiram use. The symptoms of withdrawal delirium were reported following the surreptitious administration of 600 to 900 $\mathrm{mg}$ disulfiram in one of the case. Delirium like reaction associated with disulfiram in both the cases resolved within 72 hours of stoppage of disulfiram.

\section{De novo Convulsions}

The present review identified ten cases regarding de novo convulsions associated with disulfiram use and the majority of the cases were young male clients with age ranging from 29-36 years $(\mathrm{N}=7)$. More than half of the clients were co-morbid dependent users of alcohol and tobacco $(\mathrm{N}=5)$, and half of the clients reported alcohol withdrawal seizures $(\mathrm{N}=4)$. Most of the cases reported generalized tonic-clonic seizures within 1-4 week of the initiation of $250-500 \mathrm{mg}$ disulfiram $(\mathrm{N}=8)$. However, one case reported the onset of recurrent myoclonic seizures of limbs followed by generalized tonic-clonic seizures within one week of initiation of $125 \mathrm{mg}$ disulfiram. Effective seizure control was reported by reducing the dose of disulfiram up to $125 \mathrm{mg}(\mathrm{N}=4)$ and stopping of disulfiram $(\mathrm{N}=6)$. Majority of the cases maintained alcohol abstinence with or without disulfiram use at 4 to 34 weeks follow-up $(\mathrm{N}=6)$.

\section{Reversible Hypertension}

Four cases reported disulfiram induced hypertension in which majority of the case were young male adults with age ranging from 29-39 years. In this, three cases reported de novo convulsions along with hypertension (systolic blood pressure of 170-200 and diastolic blood pressure of 100-110) within four weeks of initiation of 125-500 mg of disulfiram therapy. All the four cases have established the diagnosis of alcohol and tobacco dependence syndrome. 

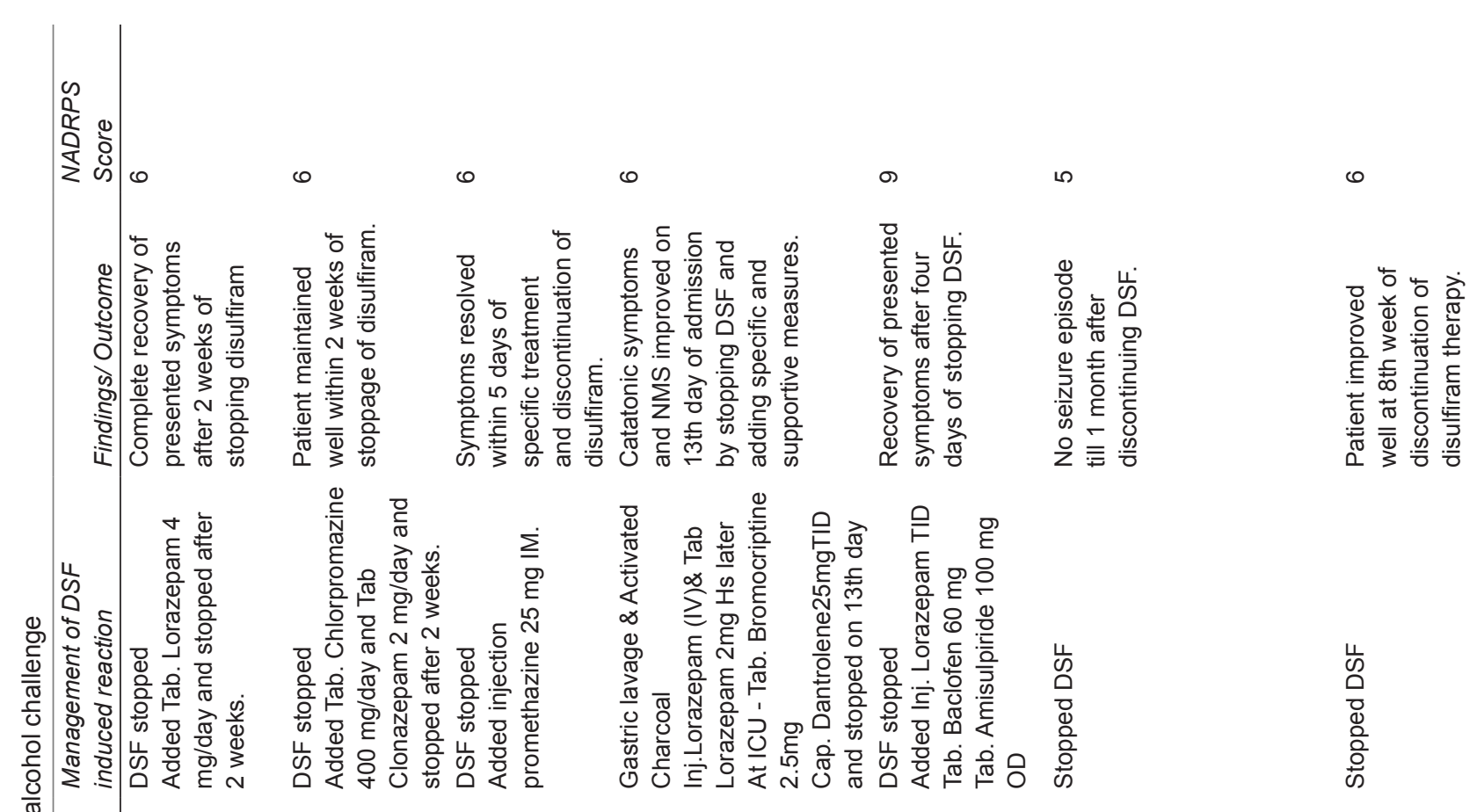

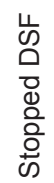
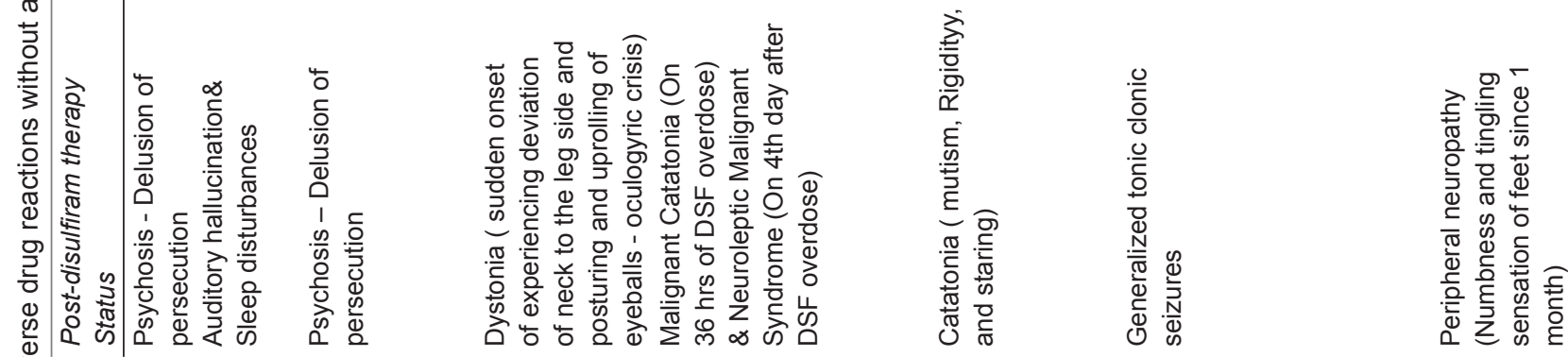

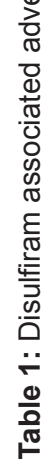

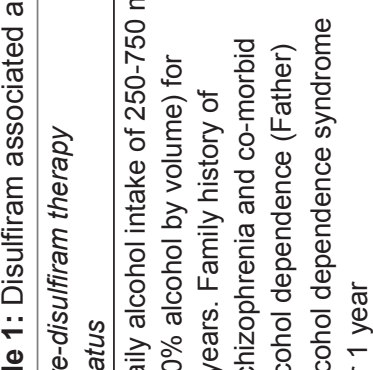

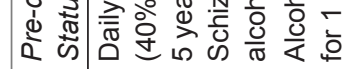

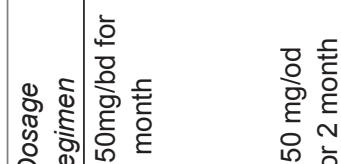

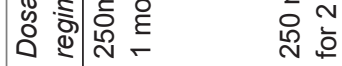

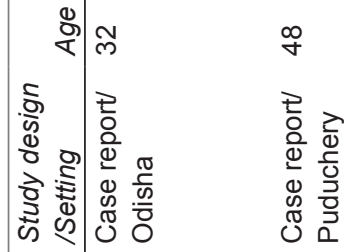

क

\begin{tabular}{|c|c|c|}
\hline 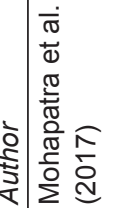 & 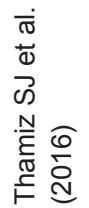 & 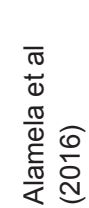 \\
\hline
\end{tabular}
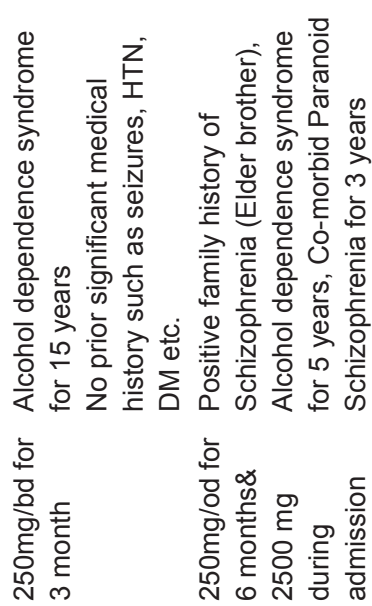

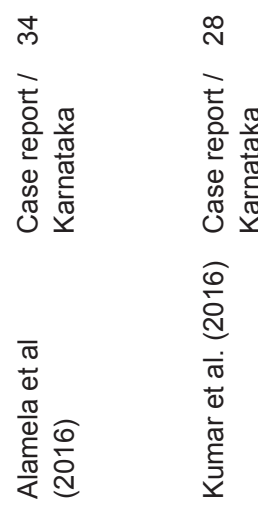
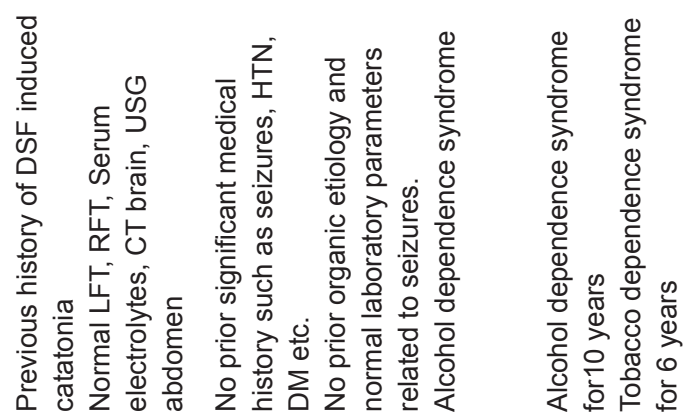

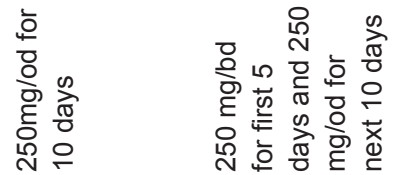

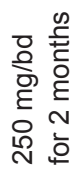

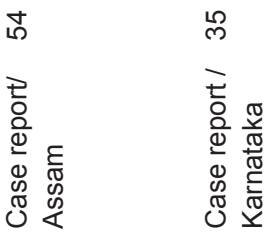

๓n

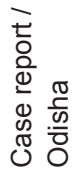

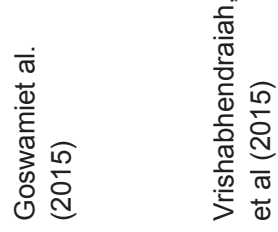




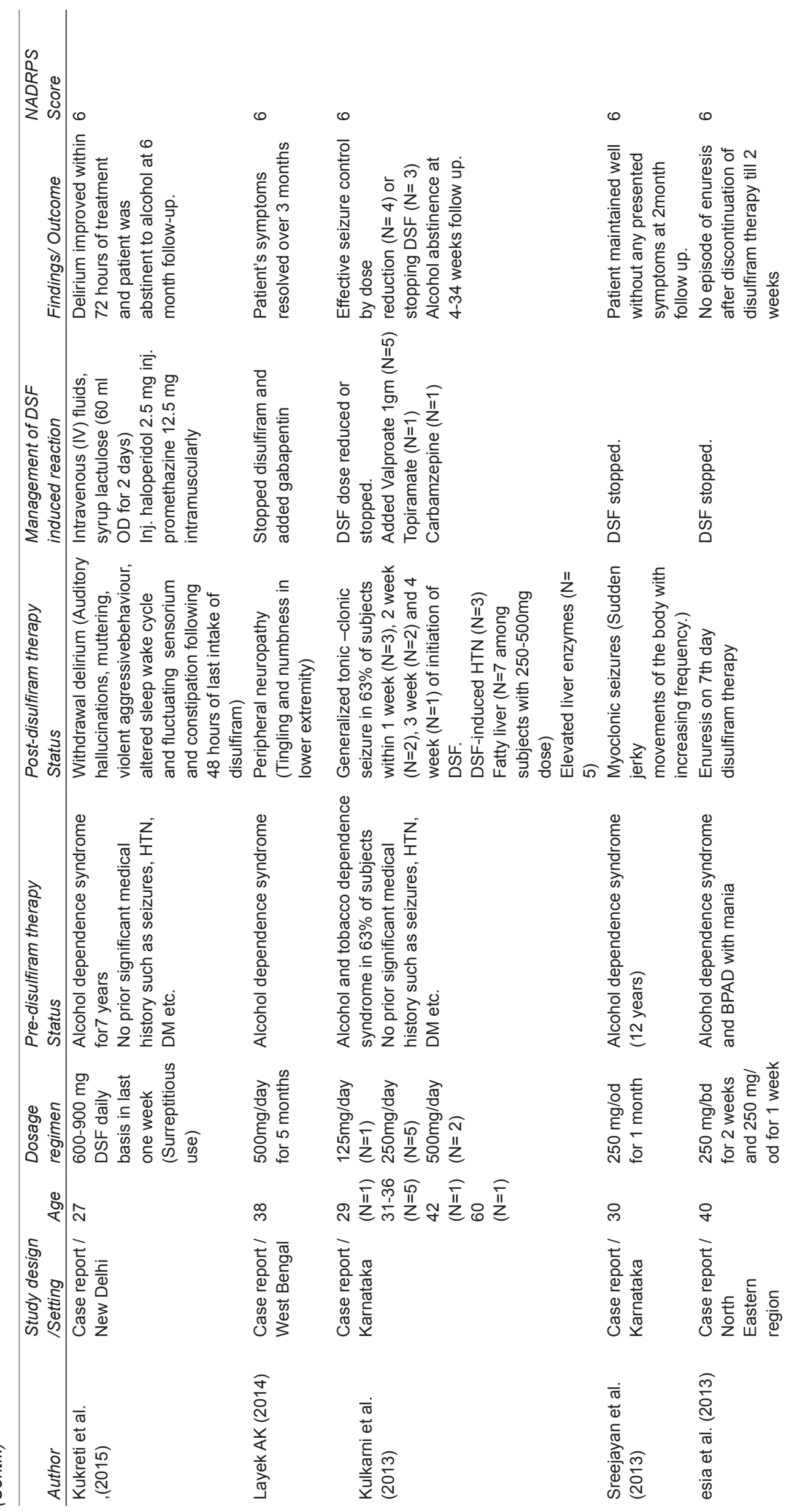

Journal of Postgraduate Medicine, Education and Research, January-March 2019;53(1):21-30 


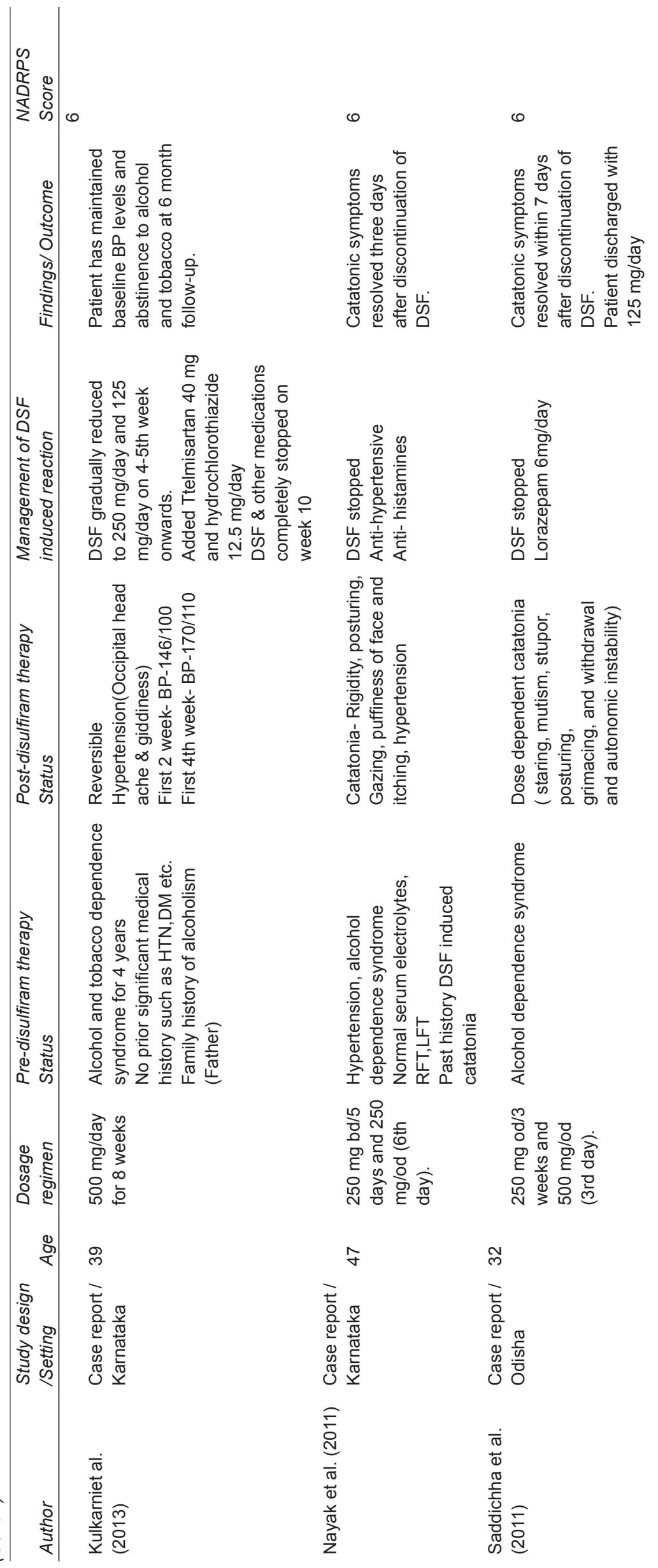




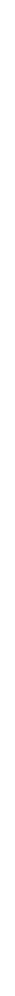

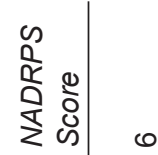

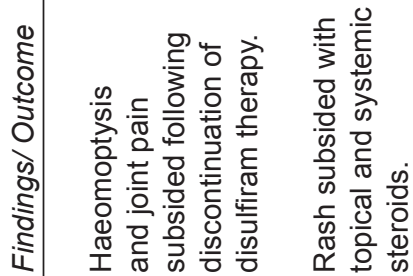

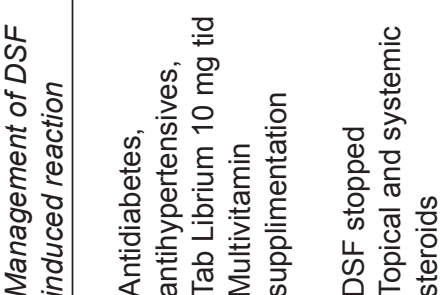

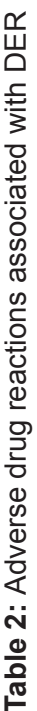

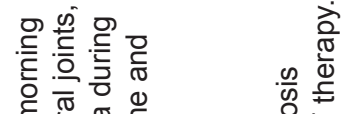

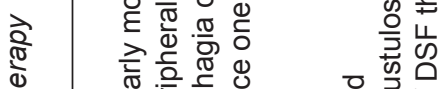

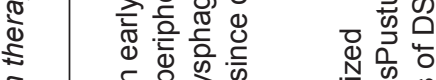

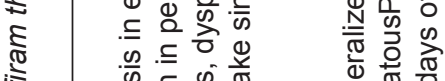

站

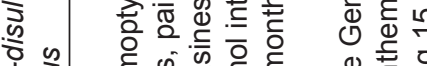

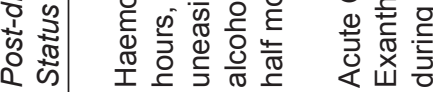

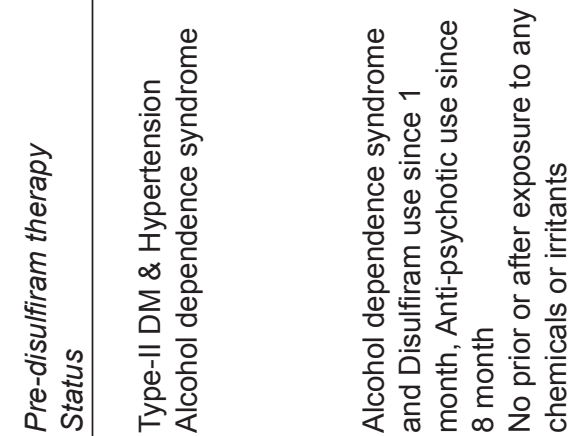

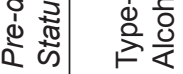

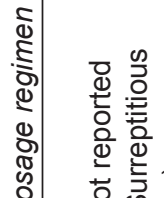

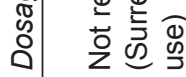

ষ

-

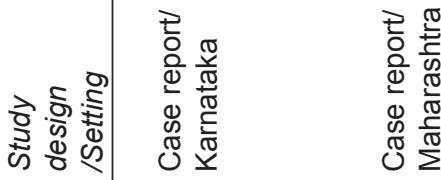

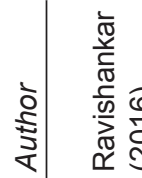

6

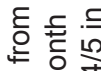

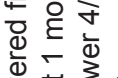

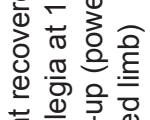

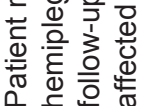

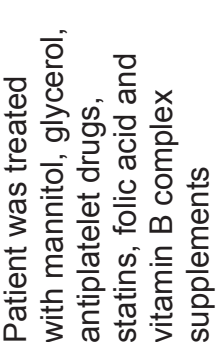

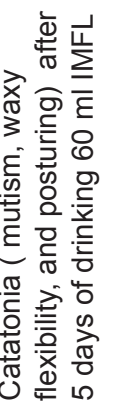

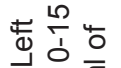

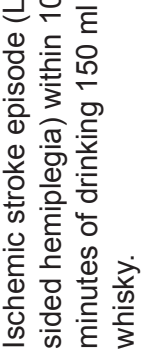

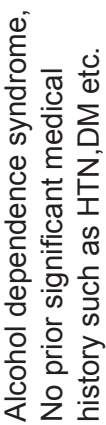

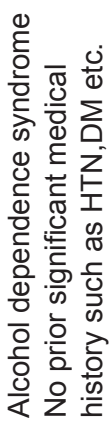

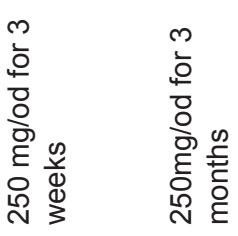

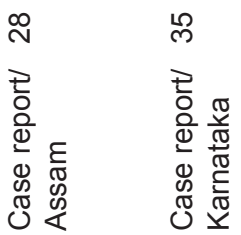

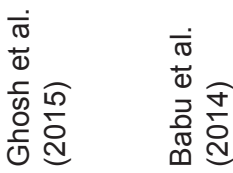

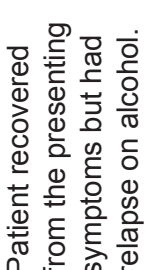

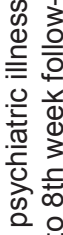

은

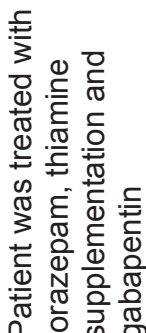

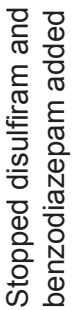
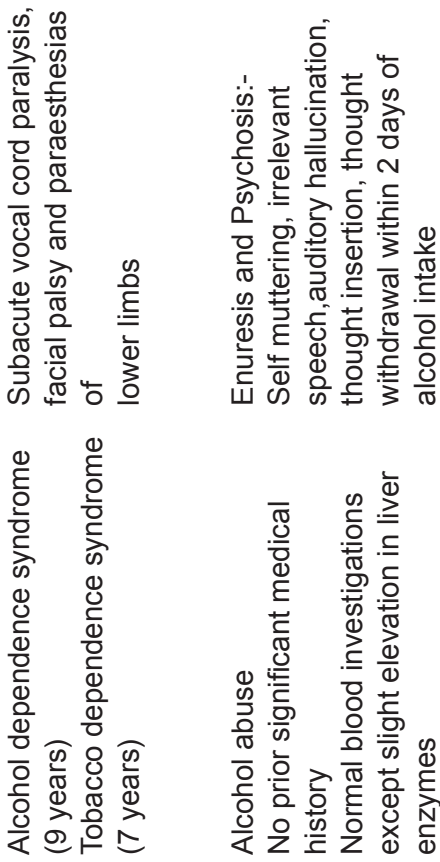

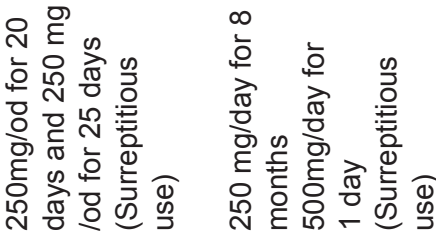

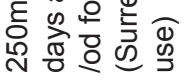

$\hat{m}$

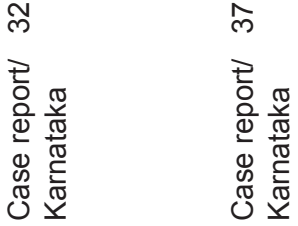

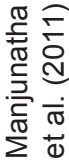

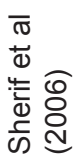


Follow-up of the cases revealed that the client has achieved baseline BP levels and abstinence to alcohol and tobacco at 6-month follow-up by adding antihypertensive and reducing doses of disulfiram.

\section{Peripheral Neuropathy}

The present review identified two cases of peripheral neuropathy within 2-5 months of initiation of $500 \mathrm{mg}$ disulfiram therapy. The symptoms of peripheral neuropathy were giddiness, bilateral joint pain, numbness and tingling sensation in feet which resolved completely within two weeks of reduced disulfiram regimen ( $250 \mathrm{mg} /$ day). However optic neuropathy reported in one of the cases on the $10^{\text {th }}$ week of disulfiram therapy which persisted even after 6 months of discontinuation of disulfiram therapy.

\section{Miscellaneous Symptoms}

A case of dystonia was developed in a client with $500 \mathrm{mg}$ disulfiram treatment for 3 months. Symptoms of dystonia resolved with specific management and stoppage of disulfiram. Enuresis was reported to a client in the first week of disulfiram therapy which resolved within two weeks of discontinuation of medication. A client with a previous history of contact dermatitis developed the same episode on the fourth day of $750 \mathrm{mg}$ disulfiram which completely resolved with specific management and without discontinuation of disulfiram therapy.

\section{Adverse Drug Reactions Associated with DER}

The present review identified six case reports of adverse drug reactions associated with the disulfiram-ethanol reaction in which disulfiram was surreptitiously administered in three cases. Haemoptysis, vocal cord paralysis and psychosis were the three reported symptoms associated with the disulfiram-ethanol reaction in which disulfiram was administered without knowledge of the client by the caregivers. All these symptoms were resolved with specific management and discontinuation of disulfiram. Catatonia and ischemic stroke episode were reported in two clients who were taking $250 \mathrm{mg}$ disulfiram for 3 weeks to 3 months respectively. Catatonia was developed after 5 days of disulfiram-ethanol reaction which resolved within a week of stopping disulfiram therapy. However ischemic stroke episode developed within 15 minutes of alcohol intake in which recovery was reported after 1 month of stoppage of disulfiram.

\section{DISCUSSION}

The present review is a systematic attempt to generate substantialdataregarding disulfiram induced adverse reactions in clients treated for alcohol dependence in the Indian setting. The results of the review specifically support the following findings; (a) psychiatric adverse events and de novo convulsions were the common adverse reactions to disulfiram treatment without alcohol challenge in this setting, (b) the magnitude of the adverse effects associated with disulfiram-ethanol reaction was largely associated with surreptitious use in this setting, (c) there is a temporal association between adverse effects and dosage of disulfiram as symptoms aggravated or abated upon increase or stoppage of disulfiram.

The review identified that psychiatric adverse effects were noted among clients who treated for alcohol dependence with $500 \mathrm{mg}$ disulfiram in this setting. Furthermore, the psychiatric symptomswere aggravated after the initiation of disulfiram therapy for clients with the previous history of psychiatric illness. Researchers noted the reporting of psychiatric adverse effects to disulfiram treatment in Indian population. ${ }^{55}$ However, the results of the review provide an evidence base for the same. Pharmacogenetic studies suggest ethnic variations regarding the effects of disulfiram in Asian population. ${ }^{56-58}$ This opens an area of discussion on the possible expansion of knowledge regarding ethnically mediated genetic differences in isozyme levels of dopamine $\$$-hydroxylase or brain acetaldehyde dehydrogenase in this setting. ${ }^{59}$

Three cases in the present review were having comorbid and family history of psychiatric illness and developed symptoms of psychosis, catatonia, and delirium after the initiation of disulfiram therapy. This further suggests the cautious use of disulfiram in clients with a history of psychiatric illness. ${ }^{60}$ The acute and chronic onset of delirium like reactions were observed in two case reports of the present review in which higher and lower doses of disulfiram therapy were attributed to the onset of the illness. Most of the previous studies suggest that delirium is associated with the disulfiram-ethanol reaction. ${ }^{61,62}$ However, both the case reports in the present review reported onset of delirium like reaction without alcohol challenge.

The review found a temporal association of adverse effects and dosage of disulfiram as symptoms aggravated or abated upon increase orstoppage of disulfiram. Catatonia, withdrawal delirium and dermatitis were the commonly reported cases with high doses of disulfiram in this setting. The above-mentioned symptoms were developed within a week of initiation of 750-2500 mg disulfiram. The findings of one case report in the present review revealed the presence of malignant catatonia with a heavy dose of disulfiram. This is in corroboration with recent findings. ${ }^{63}$ Psychotic episodes, peripheral neuropathy, and dystonia was reported within one to three months of initiation of $500 \mathrm{mg}$ disulfiram. The findings of the review also suggest that convulsions were clearly associated with the increasing dose of disulfiram as there 
were no other causes for the induction of seizures in any of the cases.

The present review identified cases of vocal cord paralysis and hemoptysis as disulfiram-ethanol reaction followed by surreptitious administration of disulfiram. Further, it was found that disulfiram was secretly administered without client's knowledge by quacks and family members. This may be due to the overemphasis on outweighing the benefits over potential risks associated with the surreptitious use of disulfiram. ${ }^{64}$ A recent study found an increasing trend of the prescribing disulfiram without informed consent by physicians and faith healers in Indian settings. ${ }^{65}$ Therefore, there is an urgent need to sensitize the healthcare professionals and the public to safeguard the ethical rights of clients in order to prevent the potentially life-threatening complications associated with the surreptitious use of disulfiram.

This review has the following limitations. The results of the review are mainly based on case reports which limit the generalization of the findings. CARE guidelines were used for assessing the methodological quality of case reports. Some assumptions have been made in NADRPS scoring due to inadequate data in available reports. The present review is a pioneer attempt for an evidence base summary of common adverse reactions and temporal association of dose-dependent adverse effects to disulfiram treatment in the Indian setting.

\section{CONCLUSION}

Psychiatric adverse events and de novo convulsions were the common adverse reactions to disulfiram treatment without alcohol challenge in this setting. Most of the adverse drug reactions were reversible with specific management and stoppage of disulfiram. The results of the review open an area of discussion regarding ethnic variations and the adverse effects of disulfiram in clients treated for alcohol dependence in this setting.

\section{REFERENCES}

1. Rehm J, Imtiaz S. A narrative review of alcohol consumption as a risk factor for global burden of disease. Subst Abuse Treat Prev Policy. 2016;11(1):37.

2. Pal A, Pattanayak RD, Sagar R. Tracing the journey of disulfiram: From an unintended discovery to a treatment option for alcoholism. J Mental Health Hum Behav 2015;20:41-43.

3. Kalra G, De Sousa A, Shrivastava A. Disulfiram in the management of alcohol dependence: a comprehensive clinical review. O J Psych. 2014;4:43-52.

4. Savas MC, Gullu JH. The disulfiram ethanol reaction: the significance of supervision. Annals of Pharmacotherapy. 1997;31:374-375.

5. Abraham J, Chandrasekaran R, Chitralekha V. A prospective study of treatment outcome in alcohol dependence from a deaddiction centre in India. Indian J Psychiatry. 1997;31:374375.

6. Prasad S, Murthy P, Subbakrishna DK, Gopinath PS. Treatment setting and follow-up in alcohol dependence.Indian J
Psychiatry. 2000;42(4):387-392.

7. Chandrasekaran R, Sivaprakash B, Chitraleka V. Five years of alcohol de-addiction services in a tertiary care general hospital. Indian J Psychiatry. 2001;43(1):58.

8. Galgali RB, Srinivasan K, Souza GD. Study of spirometry and airway reactivity in patients on disulfiram for treatment of alcoholism. Indian J Psychiatry. 2002;44:273.

9. Sidana A, Rai S, Chavan BS. Alcohol Dependence Syndrome: One year outcome studyDelhi Psychiatry Journal 2007;10(1):53-57.

10. Singh SM, Mattoo SK, Dutt A, Chakrabarti K, Nebhinani $\mathrm{N}$, Kumar S, et al. Long-termoutcome of in-patients with substance use disorders: A study from North India. Indian J Psychiatry 2008;50:269-273.

11. Chand P, Naveen CK, Murthy P, Isaac M. Addressing alcohol addiction: lessons from a hospital based audit.Indian J Med Res. 2013 Feb;137(2):394-396.

12. Balhara YP, Ranjan R, Dhawan A, Yadav D. Experiences from a community based substance use treatment centre in an urban resettlement colony in India. Journal of addiction. 2014;2014.

13. Krampe H, Ehrenreich H. Supervised disulfiram as adjunct to psychotherapy in alcoholism treatment. Curr Pharm Des. 2010;16(19):2076-2090.

14. Skinner MD, Lahmek P, Pham H, Aubin HJ. Disulfiram efficacy in the treatment of alcohol dependence: a meta-analysis. PloS one. 2014 Feb 10;9(2):e87366.

15. Crowley P. Long-term drug treatment of patients with alcohol dependence. Aust Prescr. 2015 Apr;38(2):41-43.

16. Sharma P, Murthy P, Bharath MM. Disulfiram in a "traditional" medicine sold to patients with alcohol dependence in India. Addiction. 2011;106:1870-1871.

17. Sarkar S. Surreptitious use of disulfiram. Indian J Med Ethics. 2013;10(1):71.

18. Chakraborty PK, Singh H, Divinakumar KJ. Current status of disulfiram therapy. MJAFI. 2001 Oct 1;57(4):320-321.

19. Poulsen HE, Loft S, Andersen JR, Andersen M. Disulfiram therapy-adverse drug reactions and interactions. Acta Psychiatrica Scandinavica. 1992 Nov;86(S369):59-66.

20. Gagnier JJ, Kienle G, Altman DG, Moher D, Sox H, Riley D. The CARE guidelines: consensus-based clinical case reporting guideline development. Journal of medical case reports. 2013 Dec;7(1):223.

21. Rison RA, Kidd MR, Koch CA. The CARE (CAse REport) guidelines and the standardization of case reports. 2013;7:261.

22. Naranjo CA, Busto U, Sellers EM. A method for estimating the probability of adverse drug reactions. Clin Pharmacol Ther. 1981;30:239-245.

23. De Sousa A, De Sousa A. A one-year pragmatic trial of naltrexone vs disulfiram in the treatment of alcohol dependence. Alcohol Alcohol. 2004;39:528-531.

24. De Sousa A, De Sousa A. An open randomized study comparing disulfiram and acamprosate in the treatment of alcohol dependence. Alcohol Alcohol. 2005;40:545-548.

25. De Sousa AA, De Sousa J, Kapoor H. An open randomized trial comparing disulfiram and topiramate in the treatment of alcohol dependence. J Subst Abuse Treat. 2008;34:460-463.

26. De Sousa A, De Sousa A. An open randomized trial comparing disulfiram and naltrexone in adolescents with alcohol dependence. Journal of Substance Use. 2009;13:382-388.

27. Bagadia VN, Dhawale KM, Shah LP, Pradhan PV. Evaluation of disulfiram in the treatment of alcoholism. Indian J Psychiatry. 1982;249(3):242-247. 
28. Srinivasan TN, Suresh TR, Vasantha J. Adverse effects of disulfiram and patient compliance. Indian J Psychiatry 1996; 38:47-50.

29. Murthy KK. Psychosis during disulfiram therapy for alcoholism. J Indian Med Assoc. 1997 Mar;95(3):80-81.

30. Palatty PL, Saldanha E. Status of disulfiram in present day alcoholic deaddiction therapy. Indian J Psychiatry 2011; 53:25-29.

31. Singh H. et al. Disulfiram Induced Psychosis. J Addict Depend. 2017;3(2):1-3.

32. Mohapatra S, Rath RN. Disulfiram induced psychosis. Clinical Psychopharmacology and Neuroscience. 2017;15(1):68-69.

33. Thamizh JS, Menon V, Selvakumar N, Rajkumar RP. Treatment-emergent psychosis with disulfiram in a patient with late-onset alcohol use disorder and no contributory factors.J ClinPsychopharmacol. 2016; 36(5):535-537.

34. Alamela VS, Desai M, Arunkumar C. Disulfiram-induced dystonia. J Sci Soc 2016;43:80-81.

35. Kumar KK, Bondade S, Sattar AF, Singh N. Malignant catatonia and neuroleptic malignant syndrome in relation to disulfiram overdose.Indian J Psychol Med. 2016;38(4):344-347.

36. Goswami HK, Bhuyan D, Talukdar B. Catatonia induced by disulfiram. Open Journal of Psychiatry \& Allied Sciences. 2015;6(2):143-145.

37. Vrishabhendraiah SS, Gopal Das CM, Jagadeesh MK, Mruthyunjaya N. Disulfiram-induced seizures with convulsions in a young male patient: a case study. Indian J Psychiatry 2015;57:309-310.

38. Mohapatra S, Sahoo MR, Rath N. Disulfiram-induced neuropathy: a case report. Gen Hosp Psychiatry. 2015;18(1).

39. Kukreti P, Dhiman V, Garg A. Disulfiram withdrawal delirium and non hepatic hyperammonemia: diagnostic dilemma and therapeutic challenge. Delhi Psychiatry Journal. 2015;18(1).

40. Kulkarni RR, Bairy KB. Disulfiram-induced de novo convulsions: Case series. Indian J Psychol Med. 2015;37(3):345-348.

41. Layek AK. Peripheral neuropathy: case report. Reactions Weekly. 2014;1521(1):67.

42. Sreejayan K, Praharaj, SK. Myoclonus associated with disulfiram. Journal of Neuropsychiatry and Clinical Neurosciences. 2013;25(2):37-39.

43. Tesia SS, Tesia P, Gowda MR, Preethi. Disulfiram Induced Enuresis. Asian Journal of Biochemical and Pharmaceutical Research. 2013;3(3).

44. Kulkarni RR, Bairy KB. Disulfiram induced reversible hypertension. Indian J Psychol Med. 2013;35(2):217-219.

45. Nayak V, Chogtu B, Virupaksha D, Bhandary PV. Disulfiram induced catatonia. Case Study Case Rep. 2011;1(1):6-8.

46. Saddichha S, Phutane V, Thirthalli J. De novo dose-dependent catatonia due to disulfiram therapy: a report and review. Journal of Clinical Pharmacology. 2011;51:285-286.

47. Basu D, Sharma A, Nebhinani N. Disulfiram-induced delirium. JMHHB 2009;14(2):105-107.

48. Zawar V, Nerikar S. Dermatitis recall during disulfiram therapy. Indian J Dermatol Venereol Leprol. 2004;70:33-35.
49. Ravishankar M, Rakshith N. Disulfiram induced hemoptysis: a case report. International Journal of Basic \& Clinical Pharmacology. 2016;5(1):220.

50. Jadhav PB. Disulfiram-induced toxic pustuloderma (acute generalized exanthematous pustulosis). Indian J Drugs Dermatol. 2016;2:31-32.

51. Ghosh S, Bhuyan D, Zaman UR. Catatonia associated with disulfiram therapy following disulfiram ethanol reaction. IOSR Journal of Dental and Medical Sciences (IOSR-JDMS). 2015;14(3):77-79.

52. Babu SM, Shetty CS, Aiyappa MR, Sameer S. Disulfiram Ethanol Reaction with Ischemic Stroke. Eur J Gen Med 2014;11(3):190-192.

53. Manjunatha N, Vidyendaran R, Rao MG, Kulkarni GB, Muralidharan K, John JP, et al. Subacute vocal cord paralysis, facial palsy and paraesthesias of lower limbs following surreptitious administration of disulfiram. J Neurol Neurosurg Psychiatry. 2010;81(12):1409-1410.

54. Sherif AP. Krishna Murthy K. Psychosis and enuresis during disulfiram therapy. Indian J Psychiatry. 2006;48(1):62-63.

55. Chick J. Safety issues concerning the use of disulfiram in treating alcohol dependence. Drug Safety. 1999;20:427-435.

56. Chen YC, Yin SJ. Ethnic differences of disulfiram pharmacogenetic effect in Asian population. Sy18-1. Alcohol and Alcoholism. 2014;49(1).

57. Cservenka A, Yardley MM, Ray AL. Review: Pharmacogenetics of alcoholism treatment: implications of ethnic diversity. The American Journal on Addictions. 2017;26:516-525.

58. Matsushita S, Higuchi S. Review: Use of Asian samples in genetic research of alcohol use disorders: genetic variation of alcohol metabolizing enzymes and the effects of acetaldehyde. The American Journal on Addictions. 2017;26:469-476.

59. Grover S, Basu D. The revival (or, rather, survival) of disulfiram. Addiction. 2004;99(6):785.

60. Das N, Mahapatra A, Sarkar S. Disulfiram induced psychosis: Revisiting an age-old entity. Asian Journal of Psychiatry. 2017;30: 94-95.

61. Park WC, Riggio S. Disulfiram-Ethanol Induced Delirium. Annals of pharmacotherapy. 2001;35(1):32-35.

62. Mirsal H, Yalug I, Tan D, Stern AT, Kalyoncu A, Pektas O, et al. Delirium-Associated Disulfiram and Ethanol Interactions. Prim Care Companion J Clin Psychiatry. 2005;7(5):235-237.

63. Takacs R, Milan F, Ungvari GS, Faludi G, Gazdag G. Catatonia in disulfiram intoxication - a case report and a brief overview of the literature. Neuropsychopharmacol Hung. 2016;18(2):110-114.

64. Ajish G. Ethical aspects of surreptitious use of disulfiram. Journal of Substance Use. 2016; 22(3):244-245.

65. Ajish G, Vasantmeghna MS. Jamale S. Pattern of prescription, dispensation and administration of disulfiram among patients with alcohol use disorder attending a general hospital psychiatry unit in rural India. Asian Journal of Psychiatry. 2017; 28:170-174 